Ann. Zootech., I980, 29 (1), 55-64.

\title{
Conception d'une cage à bilan adaptée à la chèvre en lactation
}

\author{
Sylvie GIGER et J. HERVIEU \\ Laboratoire de Recherches de la Chaire de Zootechnie, I.N.R.A., \\ Institut National Agronomique Paris-Grignon, \\ I6, rue Claude-Bernard, $75^{231}$ Paris Cedex o5
}

\begin{abstract}
Résumé
Les besoins nutritionnels élevés de la chèvre en lactation nécessitent une ration mixte constituée de fourrages et d'aliments concentrés. Pour mesurer la valeur nutritive de tels régimes, il est nécessaire de disposer de cages à bilan qui satisfassent aux exigences liées au comportement propre de la chèvre, à son état physiologique et à la distribution de ces deux types d'aliments. Le système décrit dans cet article est celui d'une double cage surélevée à double alimentation où la chèvre est totalement libre de ses mouvements (à l'intérieur de sa cage). Le dispositif permet une séparation et une récupération des excreta fécallx et urinaires satisfaisantes et sans contamination par les ingesta. Il ne semble pas troubler le comportement des animaux. Il a été utilisé deux années de suite pendant trois mois consécutifs par six chèvres Alpines chamoisées et a permis de tester des régimes mixtes foin-aliment concentré. Ce système qui a répondu aux objectifs fixés, devrait contribuer à une meilleure connaissance de la digestibilité et de la valeur énergétique des rations mixtes distribuées à un animal haut producteur : la chèvre laitière.
\end{abstract}

\section{Introduction}

La mesure de la digestibilité de la matière organique des aliments ou des rations complètes est à la base de l'estimation de leur valeur nutritive, énergétique en particulier. Or, un niveau élevé d'ingestion des aliments et une éventuelle interaction entre les différents composants d'une ration mixte peuvent influencer la digestibilité de cette dernière et modifier la valeur de ce type de rations couramment utilisé pour des animaux à besoin élevé de production comme une chèvre en lactation. Seule, une mesure de digestibilité sur les animaux en production permet d'apprécier ces facteurs.

Cependant, 1'utilisation de la chèvre en lactation pour ce type d'investigations se heurte à des difficultés en raison du comportement particulier de cet animal à l'auge, de sa sensibilité vis-à-vis du milieu qui l'entoure, de son état physiologique 
et de la diversité des aliments proposés. Il importe donc de concevoir un dispositif de cage de digestibilité qui soit adapté à la chèvre, tout en respectant les principes suivants pour préserver la précision des résultats :

- mesure précise des ingesta et des excreta fécaux et urinaires,

- traite des animaux en place, l'animal.

- dispositif matériel exerçant un minimum de contraintes physiques sur

Or, les dispositifs généralement utilisés ont été conçus pour le mouton mâle castré qui est l'animal choisi le plus souvent pour des études de digestibilité chez les ruminants (ChARLET-LERY, I 969 ). En effet, cet animal supporte assez bien une durée prolongée de contention et son anatomie permet une séparation relativement facile des urines et des fèces (Minson et Milford, Ig68; Schneidere et FLATT, I975).

Par ailleurs, l'utilisation de femelles pour les études de digestibilité exige la mise au point de systèmes spéciaux : sonde avec cathéter sur la vulve (ForBes et al., 1937) ou harnais (BALCh, BAR'TletT et Johnson, I951). Ceux-ci n'ont pas semblé satisfaisants pour la chèvre en raison de la sensibilité de cet animal à toute contrainte.

\section{I. - Description de la cage à bilan pour chèvres}

Cette cage a été conçue pour être utilisée par des chèvres adultes de grand format (de 50 à $70 \mathrm{~kg}$ ).

Comme le montre la photographie $I$, le système retenu est celui d'une cage double surélevée à deux dispositifs d'alimentation permettant la distribution de deux fourrages et de deux aliments concentrés. Les animaux y sont placés par couple pour éviter leur isolement. Le bloc supérieur, constitué de la cage proprement dite et du système d'alimentation, est séparé du bloc inférieur comprenant le système de récupération des fèces et des urines, par un caillebotis sur lequel se trouve l'animal situé à $0,70 \mathrm{~m}$ du sol. L'infrastructure de la cage est constituée de tubes métalliques galvanisés de $34 \mathrm{~mm}$ de diamètre et de plaques de bris aggloméré de $15 \mathrm{~mm}$ d'épaisseur.

\section{I. - La cage proprement dite}

La chèvre est totalement libre de ses mouvements dans sa cage, et seule:, deux cales en bois (o) fixées au caillebotis $(a)$ limitent son champ d'action en l'empêchant d'excréter à l'extérieur de sa case (cf. fig. I).

Compte tenu des dimensions de la cage, les excreta ne peuvent d'ailleurs tomber que sur le pourtour du caillebotis en bois de chêne recouvert d'un vernis plastifiant qui résiste à l'attaque des agents corrosifs. Une feuille de résine synthétique $(q)$ (Fibralex) est plaquée sur les parois de la cage.

La traite se fait manuellement par la porte latérale $(d)$ prévue à cet effet. 
Digestibility cage for lactating goats

Cage à bilan pour chève laitière

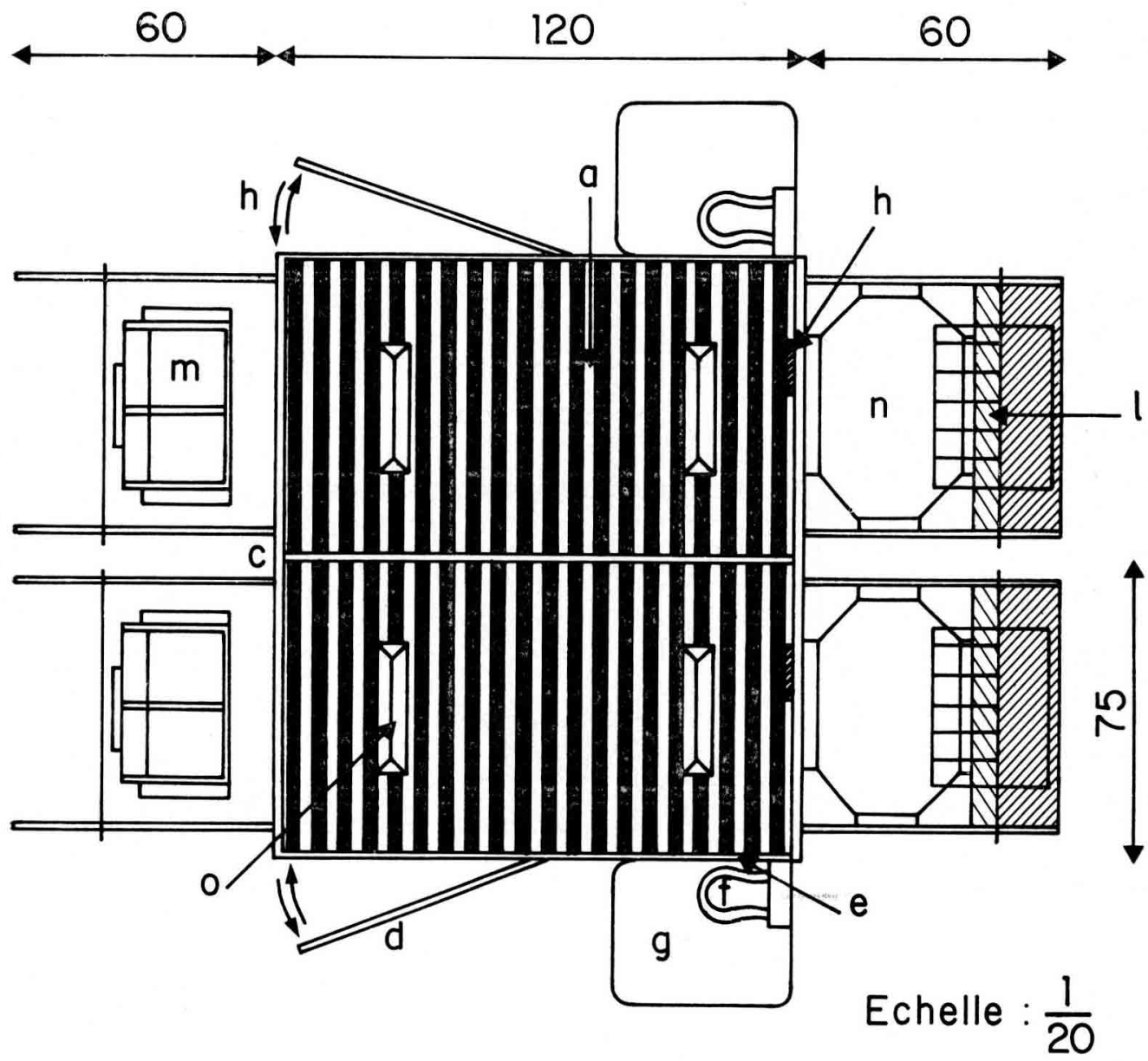

FIG. I. - Vue de dessus

a) Caillebotis (Slatted foor).

c) Cloison de séparation des cases (partition between cages).

d) Porte (door).

e) Passage de tête pour abreuvoir (Head opening to drinking bowl).

f) Abreuvoir (drinking bowl).

g) bac récupérateur des pertes d'eau (vat for recovery of water). h) Passage de tête pour mangeoire (Head opening to trough).

1) Ratelier (Feed rack).

m) Mangeoire à concentrés (Trough for concentrates).

n) Bac récupérateur des refus (vat for recovery of refusals).

o) Cales évitant la chute des excreta dans la mangeoire. (Device preventing excreta from falling into the trough). 
Digestibility cage for lactating goats

Supevelevated double cage

Cage à bilan pour chèvre laitiève

Type double surélevé

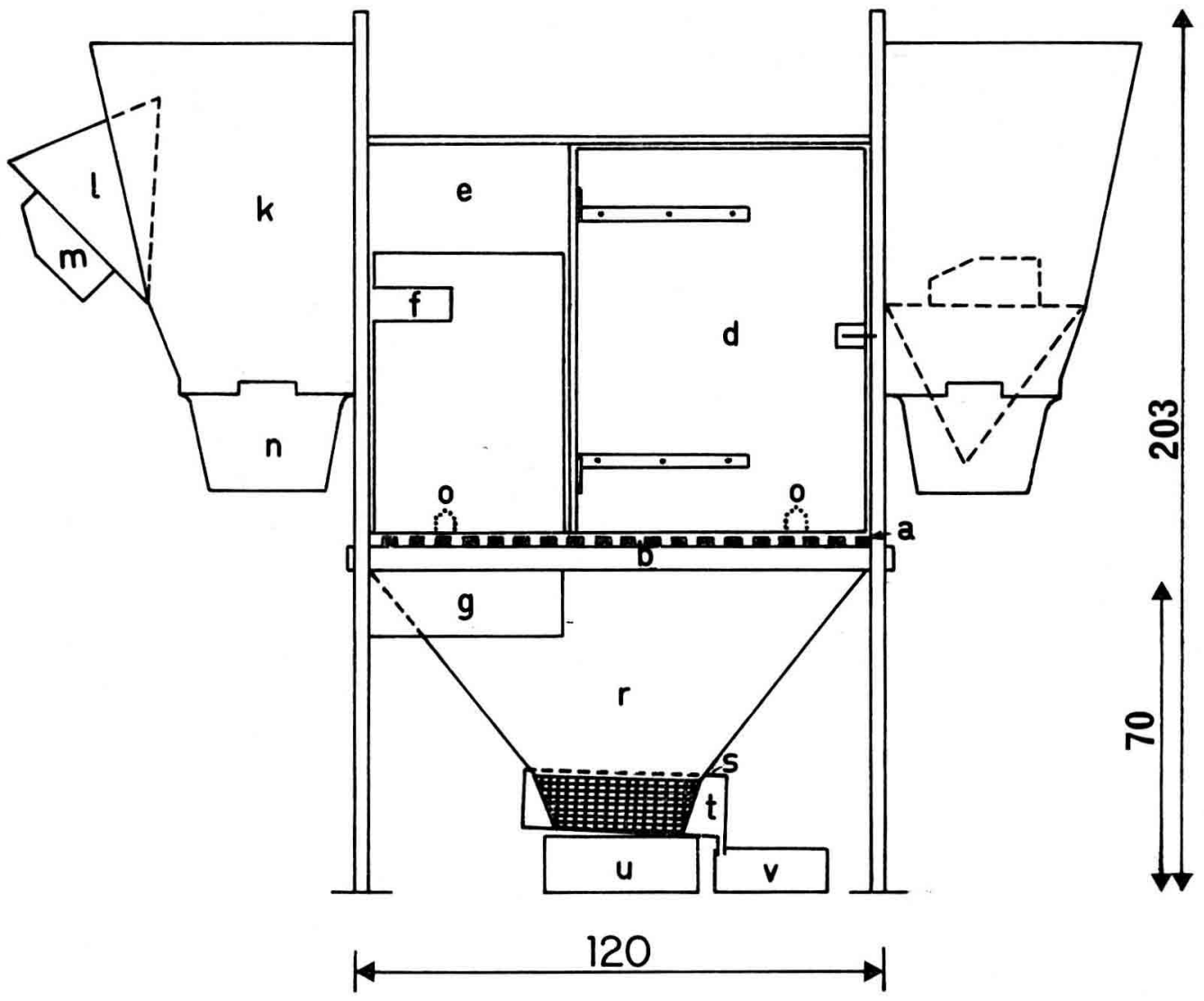

Echelle : $\frac{1}{20}$

FIG. 2. - Vue de profil (In profile).

a) Caillebotis (Slatted floor).

b) Support de caillebotis (Slatted floor support)

d) Porte (Doov).

e) Passage de tête pour abreuvoir (Head opening to drinking boull).

f) Abreuvoir (Drinking bowl).

g) Bac récupérateur des pertes d'eau (Vat for recovery of watev).

k) Mangeoire (Trough).

l) Ratelier (Feed rack). in) Mangeoire à concentrés (Trough for concentrates).

n) Bac récupérateur des refus (Vat for recovery of refusals).

o) Cales évitant la chute des excreta dans la mangeoire (Device preventing excreta from falling into the trough).

r) Cône récupérateur des excréta (Cone for recovery of excreta).

s) Tamis de séparation fèces-urine (Sicve for separating faeces from urine).

t) Gouttière canalisant l'urine (Groove for urine collection).

u) Bac à fèces (Vat for faeces).

v) Réservoir à urine (Recipient for urine). 




PIOTOGRAPHIF I

Vue d'ensemble de deux groupes de deux cages à bilan pour chèves en lactation

(cliché : Ir. Girato)

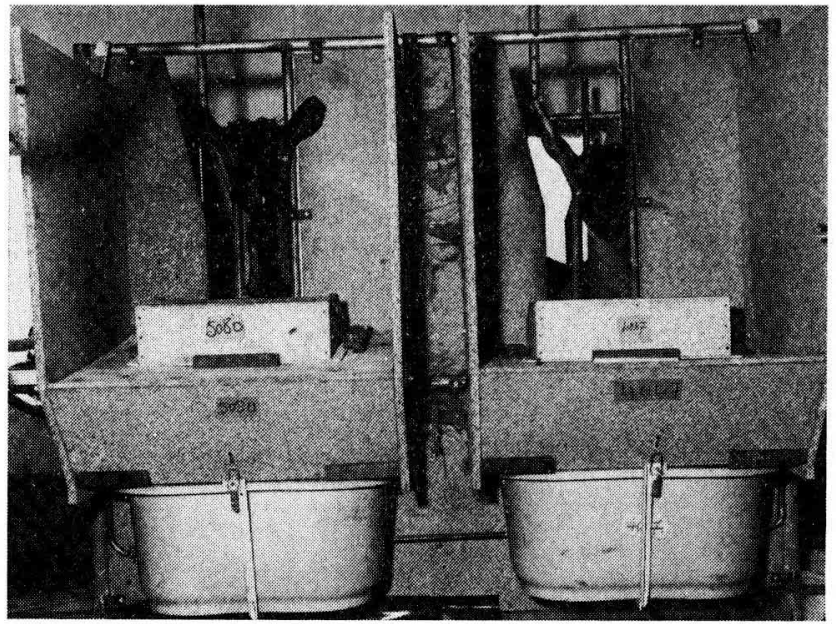

PIIOTOGRAPHIE 2

Vue de face du système de distribution des aliments et de récupération des rejus.

(cliché : H. GiratD) 


\section{2. - Le système d'alimentation}

\section{a) Système de distribution des aliments.}

Étant donné le comportement particulier de la chèvre et la nécessité de distribuer deux types d'aliments, la mangeoire décrite par HERviEU (I974) pour des fourrages a dû être modifiée. Dans sa conception actuelle, mangeoire $(m)$ et ratelier $(l)$ accolés sont basculants afin de permettre la distribution d'aliments concentrés en position abaissée (photographie 2) et de foin en position relevée (photographie I) qui est d'ailleurs la plus fréquente (fig. I et 2).

Le système de cornadis adopté ne permet pas à l'animal de gaspiller sa nourriture : en effet, il ne peut introduire ou sortir sa tête qu'au niveau de la partie supérieure $(h)$ (fig. 3), alors que les aliments ne lui sont accessibles que si elle se trouve dans le couloir de contention $(i)$ qui est plus étroit. Avec le modèle de cage utilisé, la hauteur de cette partie est réglable en fonction de la taille de chaque chèvre.

Le fourrage tiré du ratelier $(l)$ et non consommé, tombe dans un bac $(n)$ dont les dimensions ont été calculées pour que la chèvre puisse en atteindre le fond, mais qu'il permette de contenir des refus en quantité importante ainsi que le ratelier et son contenu au moment de la distribution de l'aliment concentré.

\section{b) Systeme d'approvisionnement en eau}

Un abreuvoir automatique individuel $(f)$ a été installé sur le côté de la cage (fig. I et 2). Chaque abreuvoir, à niveau constant, est relié à un réservoir étalonné qui permet de mesurer la quantité d'eau bue.

\section{3. - Recueil des fèces et des urines}

Le système de récupération et de séparation des urines et des fèces comporte plusievrs parties (fig. 2 et 3 ) :

— un cône récupérateur $(r)$ des urines et des fèces, basculant pour permettre son nettoyage quotidien,

- un tamis plastifié (s) de séparation des excréments sur lequel roulent les fèces tandis que les urines coulent au travers et sont récupérées sur une gouttière inclinée $(t)$ : les mailles de ce tamis ont $2 \mathrm{~mm}$ de côté,

- $1 \mathrm{n}$ bac de récupération des fèces (v),

- un réservoir de recueil des uines (v) d'une capacité de 5 litres.

Au niveau du caillebotis $(a)$, l'étanchéité entre la cage et le cône $(r)$ est assurée par une bande de plastique $(p)$ qui borde le bord supérieur du cône sur les quatre côtés, ce qui évite les pertes d'urine. 
Digestibility cage for lactating goats

Cage à bilan pour chivre laitière.



FIG. 3. - Coupe schimatique de face (Front soction)

a) Caillebotis (Slatted floor).

b) Support de caillebotis (Slatted foor support).

c) Cloison de séparation des cases (Partition between cages).

f) Abreuvoir (Drinking bowl)

g) Bac récupérateur des pertes d'eau (Vat for recovery of water).

h) Passage de tête pour mangeoire (Head opening to trough).

i) Couloir de contention (Restraint passage).

j) Barre de cornadis (Fed rach bar). o) Cales évitant la chute des excreta dans la mangeoire (Device preventing excreta from falling into the trough).

p) Plastique d'étanchéité (Plastic for tightness).

q) Feuille de résine pour étanchéité (Resin sheet for tightness).

r) Cône récupérateur des excreta (Cone for vecovery of excretal).

s) Tamis de séparation fèces-urine (Sieve for separating faeces from urine).

t) Gouttière canalisant l'urine (Groove for urine collection).

u) Bac à fèces (Vat for faeces).

v) Réservoir à urine (Recipient for urine). 


\section{II. - Discussion}

Ce dispositif a été utilisé deux années de suite pendant plus de trois mois consécutifs par six chèvres en lactation pour des mesures de digestibilité et de bilans azotés.

\section{r. - Utilisation de la cage}

Le système d'alimentation retenu tient compte du comportement particulier de la chèvre à l'auge (Morand-Fenr, Hervieu et Bremart LE Gousse, I977) : cet animal commence chacun de ses repas de fourrage par passer en revue et trier la nourriture offerte après l'avoir tirée du ratelier. Avec le dispositif retenu, le tri du foin est restreint par le type de ratelier et son éparpillement, limité sur les côtés par les parois latérales. La chèvre a accès au foin qu'elle a fait tomber dans le bac situé sous le ratelier. Bien que la chèvre soit en liberté dans sa cage, on ne retrouve pas de crottes dans le bac de récupération du foin grâce au système de cales en bois fixées au caillebotis.

La séparation des urines et des fèces se fait de façon très satisfaisante quand la teneur en matière sèche des fèces dépasse $35 \mathrm{p}$. Ioo, ce qui est généralement le cas. Au-dessous de ce seuil, les crottes plus humides roulent moins facilement sur le tamis, et une partie peut même rester dans la partie inférieure. Cependant, même dans ce cass, le lessivage des fèces par les urines est quasi inexistant car ces dernières ne coulent que sur une largeur de deux centimètros de chaque côté du tamis et sur une longueur de cinq centimètres lorsqu'il est nettoyé quotidiennement. Les fèces et les urines ne se mélangent pas et sont exempts de contamination par les aliments et par l'eau. L'étanchéité du système est bonne et les pertes d'urine sur les côtés de la cage sont inexistantes car aucune partie en bois brut n'est en contact direct avec de l'urine ou des fèces, ce qui évite les possibilités d'imprégnation du bois par les excréments. De par sa nature chimique, le cône retient la quasi-totalité des poils par électrostaticité.

En outre, ce dispositif présente de nombreux avantages par rapport aux dispositifs de sonde et de harnais qui perturbent souvent 1'animal : il évite les risques d'infection (ClabBY et al., I966) et ne nécessite pas de faire des récoltes trois ou quatre fois par jour (BALCH, BARTI, TT et JOHNSON, I95I), ce qui limite le temps de main-d'ouvre. Enfin, l'anatomie et la sensibilité de la chèvre la rendent mal adaptée aux harnais qui ne peuvent prendre appui sur la queue souple et mobile de cet animal.

Le plan de la cage permet d'adapter facilement un autre cornadis à 1'opposé du premier (cf. figs. I et 2 ). Avec ce dispositif, on peut offrir simultanément quatre aliments dont deux en permanence pour étudier la valeur nutritive de leur combinaison dans la ration, ce qui montre la grande souplesse d'utilisation de cette cage.

D'autre part, cette cage est entièrement démontable, ce qui permet d'utiliser à d'autres fins les bâtiments expérimentaux en dehors des périodes de mesure de digestibilité.

\section{2. - Comportement des animaux}

Au cours des différentes expériences, les animaux n'ont montré aucun trouble de comportement, même après trois mois de présence dans ces cages. Les animaux n'ont pas réduit leur niveau d'ingestion au moment de leur montée en cage et 
ont maintenu leur niveau d'alimentation et d'abreuvement. Les productions laitières ont évolué comme prévu, les chèvres se sont toujours couchées normalement et leurs aplombs n'ont pas montré de signe de fatigue. Elles semblent être bien accoutumées au système de caillebotis et ne pas être gênées par leur position surélevée. Au cours de nos observations, nous n'avons pas constaté de modification dans le comportement des animaux alors qu'un système de contention peut perturber les chèvres et influencer les résultats expérimentaux comme l'indiquent SChNEIDFR et FitaTT (I975) même s'il a été tenté de limiter son caractère contraignant (Gorski et al., I957; RAABE, I96I; Piatkowski, I963; Jentsch, I969).

\section{Conclusion}

Il n'existait pas, à notre connaissance, de description de dispositifs bien adaptés à la mesure de la digestibilité de rations complètes sur la chèvre en lactation. Le dispositif utilisé qui permet en outre la mesure des bilans azotés requiert aux exigences nécessaires à l'obtention d'une précision correcte des mesures sans modifier le comportement des animaux, tout en satisfaisant les contraintes liées à l'utilisation de femelles lactantes recevant une alimentation mixte. Ce plan de cage devrait contribuer à une connaissance plus rigoureuse de la digestibilité et de la valeur énergétique des rations mixtes distribuées à des niveaux nettement supérieurs à l'entretien, ainsi que des phénomènes de digestibilité associative entre les différents aliments chez la chèvre en lactation.

Accepté pour publication en février 1980.

\section{Summary}

\section{Conception of digestibility cages fitted to lactating goats}

The nutritional requirements of lactating goats are high and require utilization of mixed rations including hay and concentrates. For measuring the nutritive value of such rations, it is necessary to use metabolism cages fitted to the behaviout of the goat, its physiological state and the administration of two kinds of feeds. The system described in this paper consists of a superelevated double cage with 2 feeding systems in which the goats are entirely unconstrained. A satisfactory separation and a collection of urine and faeces without contamination by the feeds can be achieved in this way. The behaviour of the animals does not seem to be disturbed. The system was used for two years during three consecutive months in six Alpine goats fed mixed rations of hay and concentrates. The results obtained might contribute to a better knowledge of the digestibility and nutritive value of mixed rations given to a high yielding animal : the dairy goat.

\section{Références bibliographiques}

BAICH C. C., BARTLETT S., Johnson V. W., I95I. Apparatus for the separate collection of faeces and urine from cows. J. Agric. Sci., 41, 98-Ior.

CHARLET-LERY G., I969. Methods for determination of digestibility coefficients of feeds for ruminants. E.A.A.P. Report $n^{\circ}$ I from the study Commission on animal Nutrition, Ed. Mariendals Boktrykheri A S, Gjovik, Norway. 
Clabby J., Gibson E. H., Moss M. S., Rylance H. J., ig66. A urine collecting stall for horses. Lab. Pract., 15, 559-560.

Forbes E. B. J., BRATZLER J. W., BLACK A., BranaN W. W., 1937. The digestibility of rations by cattle and sheep. Penn. Agr. Sta. Bull, 339.

Gorski J., Blosser T. H., MURdock F. R., Hodgson A. S., SONi B. K., and ERB R. E. I957. A urine and faeces collecting apparatus for heifers and cows. J. Anim. Sci., 16, roo-rog.

Hervieu J., 1974. Discussion sur le comportement alimentaire des chèvres alimentées avec du foin exclusivement. In "Journées d'étude surl'alimentation de la chèvre laitière ", I 3 juin 1974, p. 159-16I, ed. by I.T.O.V.I.C., 149, tue de Bercy, 75579 Paris Cedex I2.

JENTSCH W., I969. Zur Technik der getrennten Eirfassung von Kot und Harn bei Kühen. Arch. Tierernähr, 19, 37I-I 74 .

Minson D. J., MiLFORD R., I968. Equipment and housing for intake and digestibility studies with large numbers of wethers. $J$. Agric. Sci., 71, 381-382.

Morand-Fehr P., Hervieu J., Bremart LE Gousse C., i977. Comportement alimentaire de la chèvre : réaction de l'animal vis-à-vis du fourrage distribué. In "Symposium sur la chèvre des pays méditerranéens ", Malaga, Granada, Murcia, 3-7 octobre 1977, p. I56-I60. Éd. FEZ, Comité espagnol.

PiatKowski B., I963. Uber das getrennte quantitative Sammeln von Kot und Harn bei Milchkiihen. Arch. Tierernähr., 13, 103-106.

RAABE R., 1968. An efficient method of excreta collection from caged sheep. Lab. Pract., 17, 2 I 7-2I8.

SCHEIDER, FLATT, I975. In "The evaluation of feeds through digestibility experiments ", p. I0-4I, published by the University of Georgia Press, Athens. 